\title{
Petit précis de théorie pour comprendre El Niño
}

\author{
Marion Saint-Lu', Julie Leloup ${ }^{2}$ \\ 1. Laboratoire des sciences du climat et de l'environnement, \\ Institut Pierre-Simon Laplace, Université Paris-Saclay, Gif-sur-Yvette \\ 2. Laboratoire d'océanographie et du climat: expérimentations \\ et approches numériques, Institut Pierre-Simon Laplace, \\ Université Pierre-et-Marie-Curie, Sorbonne Universités, Paris
}

marion.saint-lu@lsce.ipsl.fr

\section{Résumé}

EI Niño est un phénomène climatique complexe. Malgré ses multiples facettes, son existence est bien expliquée et son fonctionnement bien documenté. Depuis plusieurs décennies, la théorie relative à El Niño se constitue à mesure que les mécanismes en jeu sont mis en évidence, débattus et complétés. Nous en proposons ici une synthèse actualisée.

\section{Abstract}

\section{Essentials of theory to understand El Niño}

El Niño is a complex climatic phenomenon. Despite its multiple aspects, its existence is well explained and its physics well-documented. For a few decades, the theory related to EI Niño has been built and expanded as mechanisms are suggested, debated and further complemented. Here we present an up-to-date synthesis.
$\mathrm{T}$ ous les deux à sept ans environ, les structures climatiques du Pacifique tropical se réorganisent, avec un réchauffement de l'océan à l'est qui s'accompagne de bouleversements dans les courants, les vents, les pluies, etc. Cet état correspond à El Niño, la phase chaude d'Enso (pour El Niño-Southern Oscillation). Le Pacifique varie ainsi entre des phases « neutres », des phases chaudes El Niño et des phases froides La Niña (Philander, 1989).

Les événements El Niño et La Niña provoquent des sécheresses dans certaines régions, des inondations dans d'autres, avec des conséquences importantes en particulier dans les pays vulnérables : les épidémies sont favorisées, l'agriculture et les ressources en eau sont affectées, les écosystèmes sont touchés, etc. $\mathrm{Ce}$ phénomène touche lourdement une grande partie de la planète, ce qui a motivé les recherches visant à mieux le comprendre et l'anticiper. L'intense El Niño de 1982-1983 a été le premier événement de grande ampleur observé par satellites et dont les impacts ont été mondialement médiatisés. Cela a définitivement lancé la recherche sur le sujet avec, par exemple, la mise en œuvre d'un réseau de mesures in situ à l'échelle du Pacifique et de grands programmes internationaux dédiés (Toga-Coare).

Depuis plusieurs décennies, la théorie relative à Enso se développe et s'étoffe. Les mécanismes proposés pour expliquer l'existence et le fonctionnement d'Enso sont débattus, conciliés ou divisés selon différents points de vue. Dans la nature, ils ne sont pas exclusifs et peuvent opérer ensemble, bien que la prédominance des uns et des autres ne soit pas encore bien établie. Ici, nous présentons une synthèse non exhaustive actualisée de la théorie d'Enso (au sens de l'ensemble des idées théoriques qui se rapportent à Enso).

\section{Les différentes phases d'Enso dans le Pacifique tropical}

En conditions normales (figure 1), les alizés soufflent d'est en ouest sur le Pacifique tropical. À l'ouest, ils entretiennent la warm pool, réservoir d'eaux chaudes de températures supérieures à $28{ }^{\circ} \mathrm{C}$, nourrissant la convection atmosphérique. Cette dernière constitue la branche ascendante d'une cellule convective prenant place au niveau de l'équateur, appelée cellule de Walker. À l'est, les alizés provoquent une remontée d'eaux froides au niveau de l'équateur ou upwelling. Dans l'océan, la profondeur à laquelle la température de l'eau décroît brusquement, ou thermocline, est faible à l'est du Pacifique et plus importante à l'ouest. Le gradient est-ouest des températures des eaux de surface maintient la force et la direction des alizés et la thermocline plus profonde à l'ouest du bassin.

En conditions El Niño, les alizés faiblissent, la warm pool s'étend vers l'est, déplaçant avec elle la branche convective de la cellule de Walker et les pluies qui lui sont associées. L'upwelling faiblit et la thermocline s'aplatit. 
En conditions La Niña, les alizés se renforcent, intensifient la convection et confinent la warm pool plus à l'ouest. L'upwelling est renforcé et la pente de la thermocline accentuée.

\section{Les ondes du Pacifique équatorial}

Sous les tropiques, la circulation océanique est différente de celle des moyennes et hautes latitudes, car l'ensoleillement y est plus intense et l'influence de la rotation de la Terre plus subtile. Cette influence s'exprime comme une force s'exerçant sur les fluides océanique et atmosphérique, appelée force de Coriolis. Loin de l'équateur, celle-ci est importante et, combinée au forçage exercé par les vents, donne lieu à des circulations fermées sous forme de grands tourbillons (gyres). À mesure que l'on s'approche de l'équateur, la force de Coriolis diminue, pour finalement s'annuler à l'équateur. Alors que ses variations méridiennes sont négligeables loin de l'équateur, elles sont primordiales pour la dynamique équatoriale. En prenant en compte la variation de la force de Coriolis avec la latitude dans les équations du mouvement océanique ${ }^{1}$, linéarisées autour d'un état d'équilibre, des solutions ondulatoires sont obtenues (Matsuno, 1966) ; cela signifie que le mouvement océanique autour de l'équateur, approximativement entre $10^{\circ} \mathrm{N}$ et $10^{\circ} \mathrm{S}$, peut être décomposé comme une superposition d'ondes. Une onde consiste en une anomalie du niveau de l'eau et de la profondeur de la thermocline, associée à une anomalie de courant, qui se propage le plus souvent le long du " guide d'ondes » équatorial. Dans le cas d'une élévation du niveau de l'eau accompagnée d'un approfondissement de la thermocline, on parlera d'onde de downwelling ; dans le cas contraire, d'une onde d'upwelling.

Il existe différents types d'ondes. Celles qui jouent le plus grand rôle dans le fonctionnement d'El Niño sont les ondes de grande échelle et de basse fréquence dites « de Kelvin » et « de Rossby » (Lighthill, 1969 ; Gill et Clarke, 1974). Une onde de Kelvin est une anomalie à l'équateur qui se propage vers l'est, tandis qu'une onde de Rossby se caractérise par deux anomalies symétriques de part et
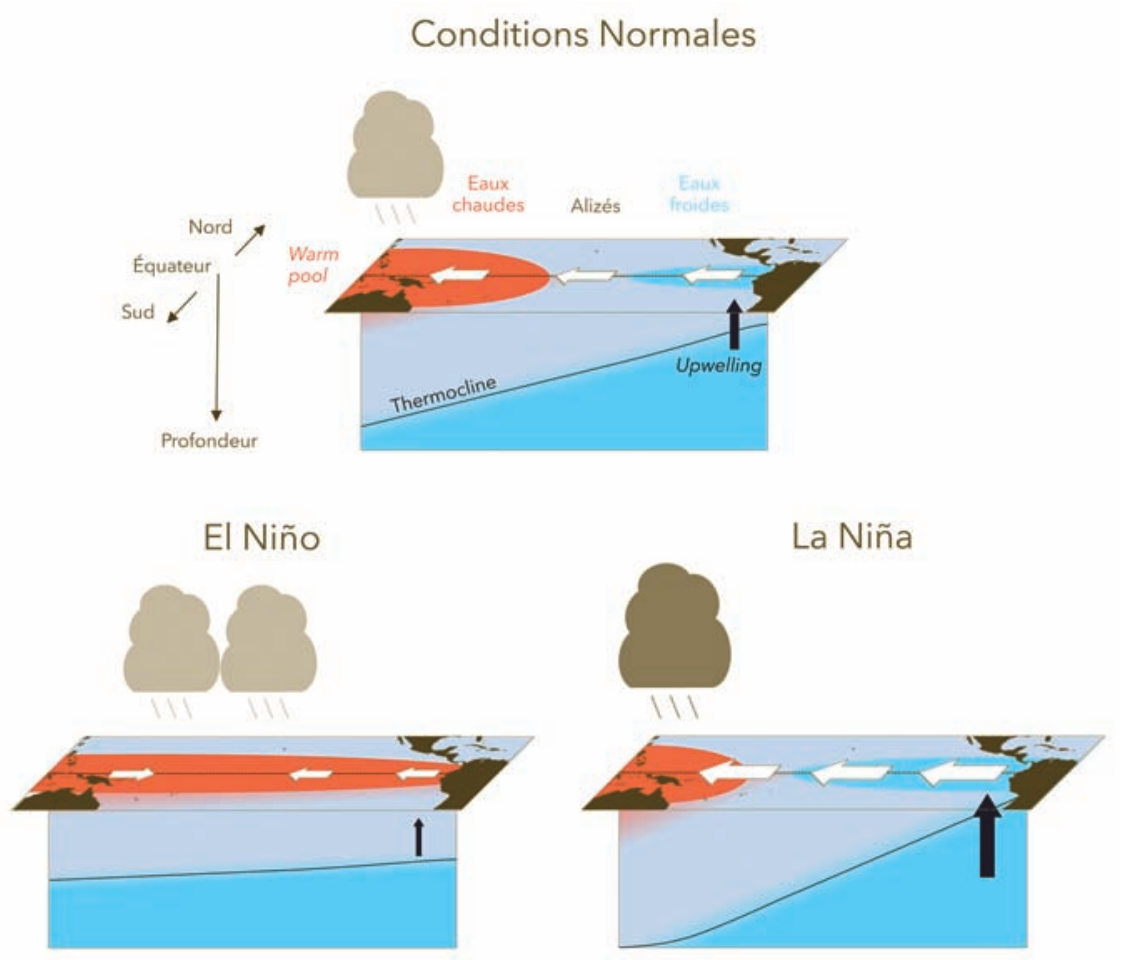

Figure 1. Schémas représentant l'état du bassin Pacifique au cours des différentes phases d'Enso. d'autre de l'équateur qui se propagent vers l'ouest. Une anomalie de vent, par exemple, engendre la formation d'ondes de Kelvin et de Rossby (figure 2).

\section{Croissance des anomalies par rétroactions positives}

Enso implique la rétroaction positive de Bjerknes (Bjerknes, 1969) : quel que soit le point de départ, un réchauffement, par exemple, en surface à l'est du Pacifique réduit le gradient zonal de température entre l'est et l'ouest du bassin, ce qui affaiblit les alizés, aplatit la thermocline et affaiblit l'upwelling équatorial forcé par le vent, induisant à son tour un réchauffement dans cette région de l'est du Pacifique, et ainsi de suite. La moindre perturbation provoquera par conséquent une réaction en chaîne, qui témoigne du fort couplage entre l'océan et l'atmosphère.

D'autres mécanismes amplifiant un réchauffement de l'est du Pacifique viennent compléter cette rétroaction de Bjerknes (Battisti, 1988 ; Dijkstra et Burgers, 2002) :

- l'affaiblissement des alizés augmente l'advection des eaux chaudes de la warm pool vers l'est du bassin et amplifie le réchauffement initial (rétroaction par advection zonale/zonal advection feedback) ;

1. Les équations du mouvement océanique traduisent les conservations de la quantitié de mouvement et de la masse.

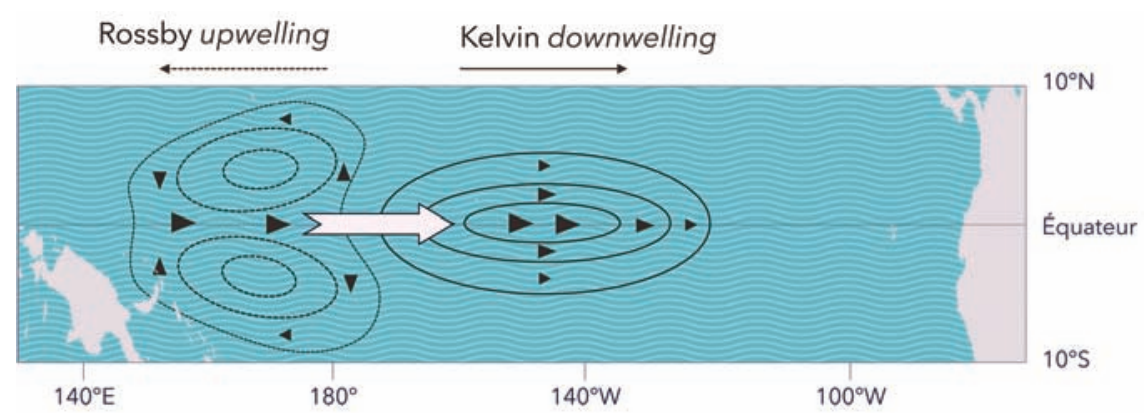

Figure 2. Anomalies de la profondeur de la thermocline (traits pleins : approfondissement; pointillés : élévation) et de courant (flèches foncées) associées aux ondes de Kelvin et de Rossby formées par un coup de vent d'ouest (flèche épaisse). Schéma adapté de Philander et al. (1984) et Boulanger (2003). 
- l'affaiblissement de l'upwelling réduit l'advection verticale des eaux froides profondes vers la surface et amplifie le réchauffement (rétroaction de l'upwelling/upwelling feedback ou Ekman feedback);

- l'approfondissement de la thermocline à l'est signifie que les eaux chaudes de surface pénètrent plus en subsurface. En d'autres termes, les eaux de subsurface remontées par l'upwelling équatorial, habituellement froides, sont plus chaudes, ce qui amplifie le réchauffement (rétroaction de la thermocline/thermocline feedback).

Par ces mécanismes, les anomalies vont croître et les événements El Niño vont pouvoir se développer. Ils sont également à l'œuvre lors d'un refroidissement de l'est du Pacifique pendant les phases La Niña.

\section{Décroissance des anomalies par rétroactions négatives}

Plusieurs rétroactions négatives sont également en jeu dans le Pacifique tropical. Elles contribuent à terminer les événements et à basculer le Pacifique vers la phase opposée. Quatre rétroactions négatives ont été proposées depuis les années 1980. Elles constituent quatre modèles d'oscillateur pour Enso : l'oscillateur retardé, l'oscillateur rechargé, l'oscillateur du Pacifique Ouest et l'oscillateur d'advection-réflexion. Pour une revue complète, on pourra se reporter à Wang et Picaut (2004).

\section{L'oscillateur retardé (The delayed oscillator)}

L'oscillateur retardé (Suarez et Schopf, 1988 ; Battisti et Hirst, 1989) repose sur la dynamique des ondes et leur réflexion sur les côtes de l'ouest (figure 3). Lors d'un événement El Niño, le relâchement des alizés est associé à une onde de Kelvin de downwelling, qui se propage et réchauffe l'est du Pacifique en y approfondissant la thermocline. L'événement El Niño s'amplifie alors par la rétroaction de la thermocline et par les autres rétroactions positives décrites précédemment. En parallèle, l'onde de Rossby d'upwelling, aussi créée par le relâchement des vents d'est, se propage vers l'ouest et se réfléchit à la côte sous forme d'onde de Kelvin d'upwelling. Celle-ci se propage vers l'est et remonte la thermocline, contrecarrant l'effet de l'onde de Kelvin initiale et contribuant à terminer l'événement El Niño. Après un certain nombre de ces réflexions, le Pacifique finit par basculer vers une phase La Niña. Il se passe environ 6 mois entre la génération de l'onde de Rossby initiale dans le centre du bassin et son retour au même point sous forme d'onde de Kelvin, ce qui est comparable à l'échelle de temps associée à la rétroaction de Bjerknes (Chang et Battisti, 1998). Lors d'un événement La Niña, par le même principe, l'onde de Rossby de downwelling associée au renforcement des alizés se réfléchit à l'ouest sous forme d'onde de Kelvin de downwelling, qui contribuera quelques mois plus tard à

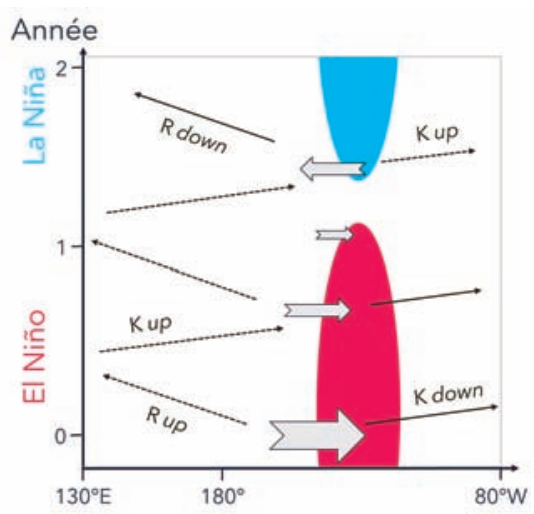

Figure 3. Mécanisme de l'oscillateur retardé. En rouge (réciproquement bleu), les anomalies chaudes (froides) de la température de surface les flèches épaisses indiquent les anomalies de vent ; les flèches fines indiquent la propagation des ondes de Kelvin (K) et de Rossby (R) upwelling et downwelling. D'après Wang et Picaut (2004). réchauffer l'est du bassin et à basculer à nouveau vers une phase El Niño, donnant ainsi lieu à une oscillation. Kessler et McPhaden (1995) ont par exemple montré que ce mécanisme expliquait la terminaison de l'événement El Niño de 1991-1992. Dans ce modèle, seule la réflexion des ondes de Rossby à l'ouest intervient. Il existe également une réflexion des ondes de Kelvin sur la côte est (sous forme d'ondes de Rossby), avec toutefois une certaine diffusion d'énergie vers les pôles. Cette réflexion à l'est est prise en compte dans le modèle de l'oscillateur d'advection-réflexion.

\section{L'oscillateur rechargé (The recharged oscillator)}

Dans le modèle de l'oscillateur rechargé (Jin, 1997), c'est un processus de décharge de chaleur qui constitue la rétroaction négative donnant lieu à l'oscillation (figure 4). Durant un événement El Niño, le relâchement des alizés crée un rotationnel de vent opposé de part et d'autre de l'équateur (car l'anomalie de vent est plus forte à l'équateur). Cela induit une divergence de masse par transport de Sverdrup ${ }^{2}$, permettant à la warm pool de se

2. Le transport de Sverdrup est lié au fait que la force de Coriolis augmente à mesure que l'on s'éloigne de l'équateur : l'effet du vent sur la déviation des courants de surface (transport d'Ekman) est alors plus fort sur la moitié du gyre la plus éloignée de l'équateur. Or, cette moitié de gyre a pour effet total de dévier les courants dans la direction opposée à l'équateur. Cela résulte au total en un transport méridien des masses d'eau dirigé vers les pôles.

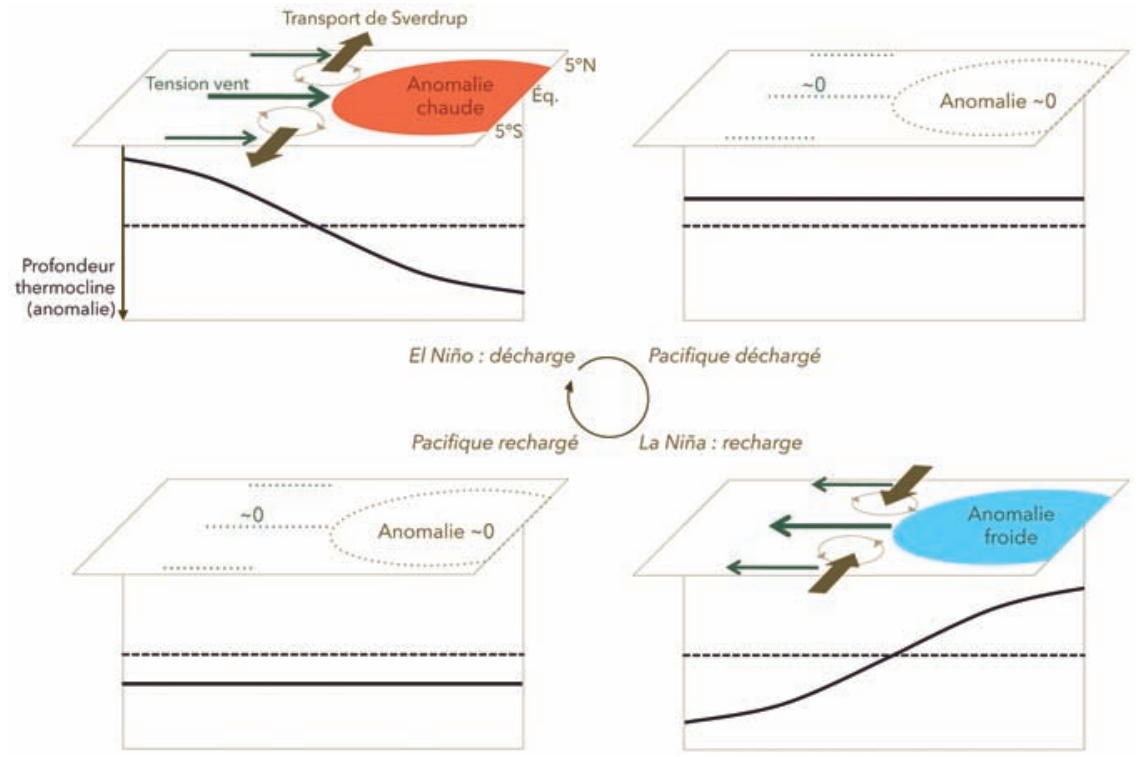

Figure 4. Mécanisme de l'oscillateur rechargé, d'après Jin (1997) et Meinen et McPhaden (2000). 
décharger en masse (et donc en chaleur). Une fois l'océan déchargé, la thermocline est moins profonde sur l'ensemble du bassin, l'upwelling équatorial est renforcé, ce qui est propice au développement d'un événement La Niña. Par les mécanismes inverses, la phase La Niña recharge l'océan, approfondit la thermocline et mène ainsi au développement d'un événement El Niño. On peut citer par exemple Meinen et McPhaden (2000), qui ont identifié les phases de recharge et décharge associées à Enso dans les observations.

\section{L'oscillateur du Pacifique Ouest (The Western Pacific oscillator)}

L'oscillateur du Pacifique Ouest (Weisberg et Wang, 1997) repose sur la formation d'anomalies de vent d'est à l'ouest du Pacifique (figure 5). Durant la phase mature d'El Niño, l'onde de Rossby d'upwelling se propage vers l'ouest, faisant remonter la thermocline de part et d'autre de l'équateur. À l'ouest du Pacifique, elle refroidit la surface et les basses couches atmosphériques, créant ainsi deux anomalies de haute pression dans l'atmosphère et donc deux anticyclones. Ces anticyclones induisent une anomalie de vent d'est à l'équateur, qui elle-même donne naissance à une onde de Kelvin d'upwelling. En se propageant vers l'est, elle donne lieu à une oscillation par le même principe que celui de l'oscillateur retardé.

\section{L'oscillateur}

\section{d'advection-réflexion (The advective-reflective oscillator)}

L'oscillateur d'advection-réflexion (Picaut et al., 1996, 1997) prend en considération les anomalies de courant océanique associées aux ondes de Kelvin et de Rossby, ainsi que la réflexion de ces ondes aux côtes (figure 6). Les événements El Niño sont associés à un décalage vers l'est du bord est de la warm pool. Des anomalies de courant vers l'est sont associées aux ondes de Kelvin (downwelling) et de Rossby (upwelling) pendant la phase El Nino. Elles contribuent à l'étalement de la warm pool. L'onde de Kelvin d'upwelling issue de la réflexion de l'onde de

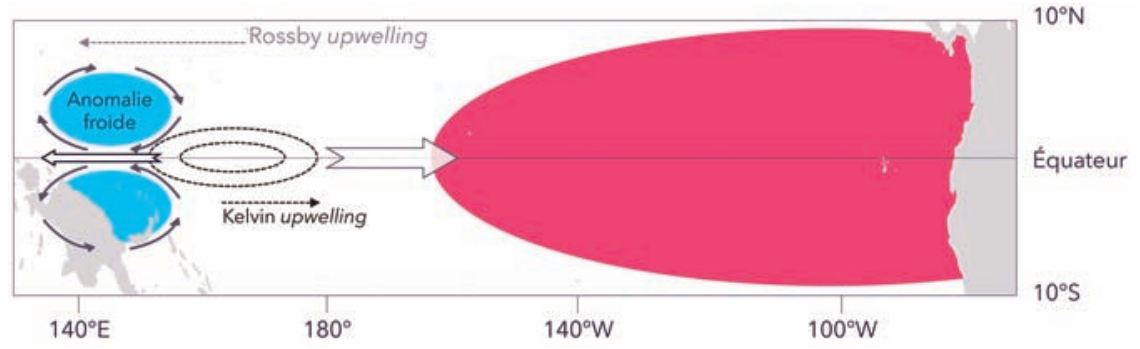

Figure 5. Mécanisme de l'oscillateur du Pacifique Ouest (même légende que les schémas précédents). D'après Wang et Picaut (2004).

Rossby à l'ouest et l'onde de Rossby de downwelling issue de la réflexion de l'onde de Kelvin à l'est sont toutes deux associées à des anomalies de courant vers l'ouest (les courants associés aux ondes changent de sens après réflexion). Ces anomalies viennent renforcer les courants moyens pour ramener la warm pool à sa position initiale, puis permettent le basculement vers la phase La Niña. Lors de l'événement El Niño de 1986-1987, l'onde de Rossby résultant de la réflexion à l'est du bassin a été observée et aurait causé le basculement vers l'événement La Niña de 1988-1989 (Picaut et Delcroix, 1995).

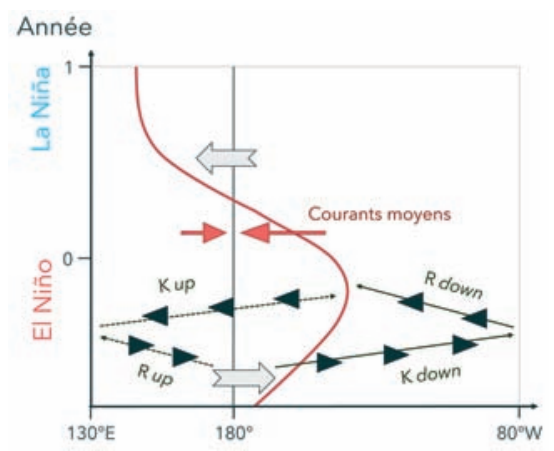

Figure 6. Mécanisme de l'oscillateur d'advectionréflexion. La ligne rouge indique le bord est de la warm pool (même légende que les schémas précédents). D'après Wang et Picaut (2004).

Wang (2001) suggère que ces différentes rétroactions peuvent opérer ensemble dans la nature et leur importance relative varier selon les événements ; il formule la théorie de l'oscillateur unifié (The unified oscillator).

Plus récemment, Clarke (2008) suggère de repenser ces modèles d'oscillateur en mettant l'accent sur l'importance de l'anomalie de température au niveau du bord est de la warm pool, plutôt qu'à l'est du Pacifique. En effet, bien que l'anomalie de température soit plus importante à l'est, la température totale n'y est pas suffisante pour déclencher la convection profonde atmosphérique. Clarke (2008) soutient que c'est pourtant la convection au centre du bassin qui va jouer le rôle clé dans le développement et la terminaison des événements, car elle est associée à un très fort couplage entre l'océan et l'atmosphère. Lors d'un événement El Niño, le déplacement vers l'est du bord est de la warm pool entraîne avec lui la convection et les précipitations. On observe en effet une anomalie convective importante au centre du bassin. Par dégagement de chaleur latente, la colonne atmosphérique chauffe et la convection s'intensifie. Les observations montrent que la réponse du vent en surface consiste en une anomalie de vent d'ouest à l'ouest de l'anomalie, qui nourrit la convection. Le point crucial ici est cette asymétrie dans la réponse du vent zonal. En effet, d'après le modèle de Gill (1980), la réponse du vent à un chauffage atmosphérique consiste en une anomalie de vent d'est à l'est de la zone de chauffage, associée à une onde de Kelvin atmosphérique (pendant de l'onde de Kelvin océanique), qui s'étale sur une grande partie du bassin, ainsi qu'en une anomalie de vent d'ouest à l'ouest, associée à une onde de Rossby atmosphérique, qui se concentre sur une région plus restreinte. La dimension spatiale de l'onde de Kelvin atmosphérique est de l'ordre de $5000 \mathrm{~km}$ du nord au sud, ce qui est bien plus grand que la dimension de l'anomalie convective associée à El Niño, de l'ordre du millier de kilomètres du nord au sud. Cette dernière est donc trop petite pour exciter l'onde de Kelvin atmosphérique. C'est alors principalement une anomalie de vent d'ouest qui est générée en réponse à l'anomalie convective. Cette anomalie de vent d'ouest engendre une anomalie de courant qui va déplacer le bord est de la warm pool plus loin vers l'est et 
intensifier ainsi le phénomène. Pour terminer l'événement, les ondes de Kelvin d'upwelling provenant de l'ouest du bassin, associées à des anomalies de courant vers l'ouest, contribuent à ramener la warm pool à sa position initiale. Par ailleurs, la remontée de la thermocline associée au processus de décharge refroidit la surface, amortissant la convection. L'approche de Clarke (2008) regroupe les différents modèles d'oscillateurs, mais l'anomalie de température à l'est du Pacifique n'entre pas en jeu dans le mécanisme. Ainsi, les modèles d'oscillateur peuvent être revus, complétés, et la théorie d'Enso est en perpétuelle évolution.

Tous ces éléments expliquent pourquoi le Pacifique tropical n'est pas dans un état stationnaire. Sa variabilité naît du fort couplage entre l'océan et l'atmosphère qui peut rendre instable une perturbation initiale, de la grande taille du bassin qui induit un délai de réponse aux perturbations, et des mécanismes de rétroactions positives et négatives qui induisent une succession de phases El Niño et La Niña. On retrouve cette variabilité interannuelle du Pacifique dans les observations : la fréquence de variations dominante de la température de surface à l'est du Pacifique a une période de 3 à 5 ans (Neelin et al., 1998, et ses références).

Cependant, Enso tel qu'on l'observe est loin d'être une oscillation interannuelle régulière entre des phases El Niño et La Niña. Ces modèles théoriques sont appelés " oscillateurs », car ils prennent en compte des processus de rétroactions positives et négatives qui donnent lieu à une oscillation dans un système simplifié. Dans la nature, cela n'implique pas nécessairement l'existence d'une oscillation auto-entretenue sousjacente. Quoi qu'il en soit, les rétroactions négatives peuvent permettre d'expliquer la terminaison de certains événements. En outre, les phases El Niño se présentent plutôt sous la forme d'événements " verrouillés » sur le cycle saisonnier (ou annuel), c'est-à-dire qu'ils débutent au printemps boréal, se développent en été, atteignent leur amplitude maximale en hiver et s'évanouissent au printemps suivant. Les modèles d'oscillateur ne tiennent pas compte de l'existence du forçage des saisons, qui va pourtant perturber le cycle interannuel et déterminer le verrouillage des événements sur le cycle saisonnier.

\section{Interaction avec le cycle saisonnier}

Zebiak et Cane (1987) ont développé un modèle simplifié pour mener une expérience dans laquelle l'état de base ne varie pas, retirant ainsi artificiellement le forçage saisonnier (voir Delcroix, 1998, pour de plus amples informations sur le cycle saisonnier du Pacifique tropical). Sans ce cycle annuel, une oscillation Enso périodique de 47 mois est obtenue (pouvant varier selon les conditions moyennes imposées), ce qui se situe bien dans l'intervalle donné par les observations (3-5 ans). Avec ce cycle annuel, Enso perd sa périodicité. L'interaction avec le cycle saisonnier est donc une source d'irrégularité d'Enso. Ce résultat est lié au fait que certaines saisons sont plus propices que d'autres au développement des événements (Philander, 1985 ; Zebiak et Cane, 1987 ; Spencer, 2004). Les anomalies vont généralement apparaître au printemps boréal, lorsque l'est du Pacifique est le plus chaud, rendant le processus de convection plus sensible aux variations de température. Elles vont ensuite pouvoir grossir en été, lorsque l'upwelling équatorial est fort, rendant la température de surface plus sensible aux perturbations de la thermocline (entre autres). De manière générale, l'été est la saison à laquelle le couplage entre l'océan et l'atmosphère (et donc la sensibilité de l'un à l'autre) est le plus fort. Le réchauffement atteindra son maximum en hiver dès lors que le couplage entre l'océan et l'atmosphère diminuera de nouveau (Zebiak et Cane, 1987) et que les alizés se renforceront. Ainsi, le forçage saisonnier contraint également la terminaison des événements. Cela n'empêche pas les rétroactions négatives des modèles d'oscillateur de jouer un rôle à la fois dans la terminaison des événements et dans la transition entre les phases, par leur effet mémoire.

Le forçage saisonnier explique aussi pourquoi les événements sont verrouillés sur le cycle annuel, c'est-àdire pourquoi ils débutent à peu près à la même période de l'année, atteignent leur maximum d'intensité autour de l'hiver et durent environ une année.

Les mécanismes que nous avons exposés jusqu'ici ignorent l'existence des aléas atmosphériques qui vont pourtant jouer un rôle perturbateur important.

\section{Rôle des aléas atmosphériques}

Les « coups de vent d'ouest » (ou WWB pour westerly wind burst) (voir Lengaigne et al., 2004) sont identifiés comme des déclencheurs potentiels d'événements El Niño. De tels coups de vent se produisent à l'ouest du bassin Pacifique, le plus souvent en hiver boréal. Ils peuvent déclencher une onde de Kelvin de downwelling qui atteindra 1'est du bassin 2 à 3 mois plus tard, y approfondira la thermocline et y déclenchera potentiellement un réchauffement par les diverses rétroactions positives. Dans le même temps, le coup de vent d'ouest force des courants qui déplacent le bord est de la warm pool vers l'est, déplaçant la convection atmosphérique et induisant une anomalie de vent d'ouest par les mécanismes déjà évoqués. Cette anomalie pourra se traduire par d'autres coups de vent d'ouest subséquents, cruciaux pour faire réagir le Pacifique. Par exemple, l'absence de ces autres coups de vent d'ouest en 2014 aurait joué un rôle important dans le non-développement de l'événement El Niño pourtant attendu (Menkes et al., 2014). Le déclenchement d'un événement El Niño par de tels coups de vent d'ouest va dépendre de l'état initial dans lequel se trouve le Pacifique. L'effet des coups de vent d'ouest sur la température de surface de l'autre côté de l'océan ne résulte pas uniquement de l'approfondissement de la thermocline associé à l'onde de Kelvin, mais également d'un jeu complexe d'advection des anomalies de température (Belamari et al., 2005). Une série de coups de vent d'ouest a ainsi déclenché le très fort El Niño de 1997-1998 (van Oldenborgh, 2000). Il en est probablement de même pour l'événement récent (2015-2016) d'amplitude comparable (L'Heureux, 2015).

Puisqu'une perturbation atmosphérique peut déclencher un événement, le forçage atmosphérique est une autre source d'irrégularité d'Enso. De manière plus générale, l'importance du forçage stochastique de l'atmosphère dans l'existence d'Enso et son irrégularité a été soulignée dans de nombreuses études (Penland et Sardeshmukh, 1995 ; Chang et al., 1996 ; McPhaden, 1999 ; Takayabu et al., 1999 ; Moore et Kleeman, 1999 ; Roulston et Neelin, 2000 ; Vecchi et Harrison, 2000 ; Chen et al., 2015). 
Dans la nature, il a été observé que les événements peuvent aussi débuter par une anomalie de température à l'est qui se propage vers l'ouest, ou une anomalie au centre qui se propage vers l'est. Jusqu'ici, nous ne nous sommes pas attardés sur la propagation des anomalies, ni sur leur étalement spatial. Ce point est déterminant pour le déclenchement, le développement et la structure spatiale des événements.

\section{Propagation des anomalies de température}

Les interactions thermodynamiques entre l'océan et l'atmosphère et les advections de chaleur peuvent conduire à l'amplification et à la propagation d'anomalies de température (Gill, 1985 ; Hirst, 1986, 1988 ; Neelin, 1991 ; Wang et Weisberg, 1996). En guise d'exemple, considérons une anomalie chaude en surface à l'est du bassin. La figure 7 décrit comment, par un jeu d'advection de chaleur, cette anomalie se propage vers l'ouest (Neelin et al., 1998 ; Philander et Fedorov 2003 ; Wang et Picaut 2004)3. Le vent en surface converge sur la zone de réchauffement, entraînant une anomalie de vent d'ouest sur l'océan et une anomalie de vent d'est sur le continent (figure 7, étape 2). Cette dernière se produisant au-dessus des terres n'a pas d'effet sur la propagation du réchauffement (Neelin et al., 1998). Au-dessus de l'océan, l'anomalie de vent d'ouest entraîne les eaux chaudes de la warm pool vers la zone de réchauffement et induit un approfondissement de la thermocline (downwelling) sur son flanc ouest (figure 7, étape 3 ). Ces deux effets amplifient le réchauffement à l'ouest de l'anomalie chaude initiale, la propageant ainsi vers l'ouest. La même réaction se produit alors sur le flanc ouest de la nouvelle zone de réchauffement agrandie (advection d'eaux chaudes et downwelling), la propageant davantage vers l'ouest (figure 7, étape 4), et ainsi de suite.

Un certain nombre d'événements débutent par une anomalie chaude à l'est qui se renforce en s'étendant vers l'ouest (Rasmusson et Carpenter, 1982 ; McPhaden, 1999). Le mécanisme

3. Ces modes de propagation ont été appelés " modes de SST (sea surface temperature) 》 (Neelin, 1991). précédent peut jouer dans cette expansion, mais l'anomalie est aussi amplifiée par les diverses rétroactions positives.

À l'inverse, certains événements, comme ceux de 1982, 1986 et 1991 (Wang, 1995), débutent par une anomalie de température au centre du bassin, qui se propage ensuite vers l'est. Harrison et Schopf (1984) ont montré qu'un front d'ondes de Kelvin de downwelling généré par des coups de vent d'ouest avait provoqué en 1982 un premier réchauffement dans le centre du bassin, précédant le réchauffement à l'est. L'effet d'une onde de downwelling est de réchauffer la surface là où la thermocline est peu profonde, donc à l'est du bassin. Cependant, si le gradient est-ouest de température est assez fort (comme c'est le cas en été), les anomalies de courant d'ouest associées au passage de l'onde transportent les eaux chaudes de la warm pool vers l'est, créant ainsi un premier réchauffement bien visible au centre du bassin.

Ces quelques exemples donnent une idée des mécanismes d'advection et d'interactions thermodynamiques qui peuvent entrer en jeu dans la propagation des anomalies de température et le développement des événements. Ces processus peuvent être indépendants de la dynamique des ondes équatoriales de Kelvin et de Rossby. Ils permettent d'expliquer les différences qu'il peut y avoir d'un événement à l'autre dans le développement de l'anomalie chaude et contribuent ainsi fortement à leur grande diversité. En effet, chaque événement est unique, du fait du grand nombre de facteurs d'influence en jeu.

\section{Et La Niña dans tout ça ?}

Les mécanismes que nous avons présentés ne s'appliquent pas toujours à l'inverse pour les événements La Niña. Ces derniers sont généralement d'amplitude plus faible que les événements El Niño (Burgers et Stephenson, 1999), ce qui peut être expliqué par un certain nombre d'asymétries, ou non-linéarités, dans les mécanismes (Choi et al., 2013 ; Im et al., 2015). Par exemple, la sensibilité de

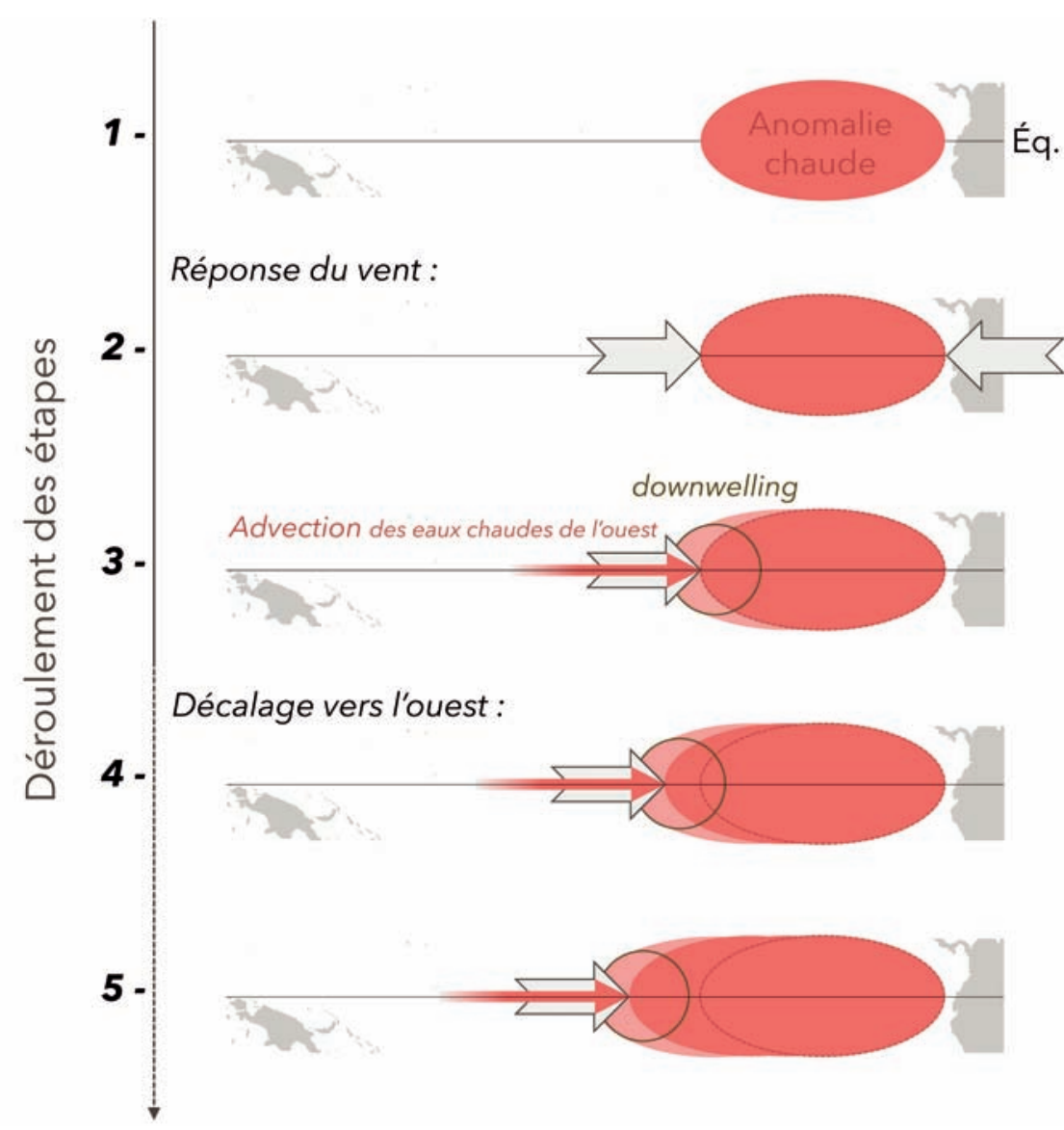

Figure 7. Exemple de propagation d'une anomalie chaude à l'est du bassin. La légende est la même que pour les autres schémas (avec une flèche rouge supplémentaire pour indiquer l'advection des eaux chaudes). On peut ajouter qu'à l'étape 2 Neelin et al. (1998) précisent que l'anomalie de vent générée à l'est de l'anomalie chaude se produit au-dessus des terres et n'a pas d'effet sur le mode de propagation. 
la convection atmosphérique à une anomalie de température n'est pas la même selon qu'il s'agit d'une anomalie chaude ou froide. En outre, les anomalies associées aux événements se superposent sur un état de base déjà bien marqué (gradient est-ouest de température, alizés, etc.), ce qui implique qu'elles n'auront pas les mêmes effets selon leur signe, pouvant être amplifiées ou amorties par cet état de base. Différentes sources de non-linéarités sont invoquées aujourd'hui dans la littérature, sans que l'on connaisse la contribution réelle de chacune. Plusieurs études mettent en particulier l'accent sur l'asymétrie dans la réponse du vent à des anomalies de température de signe opposé, en lien avec le déplacement du bord est de la warm pool et le déclenchement des coups de vent d'ouest (Kessler et Kleeman, 2000 ; Philip et van Oldenborgh, 2009 ; Choi et al., 2013).

Par ailleurs, El Niño est généralement plus court que La Niña (Larkin et Harrison, 2002 ; Kessler, 2002 ; Okumura et Deser, 2010). Certains événements La Niña sont déclenchés par la terminaison d'événements El Niño très forts ${ }^{4}$ (Larkin et Harrison, 2002 ; Lengaigne et al., 2006 ; Vecchi et Harrison, 2006 ; Vecchi, 2006 ; Okumura et Deser, 2010). Ces asymétries sont également liées aux non-linéarités dans les processus de rétroactions positives/négatives, qui sont généralement plus intenses lors des événements El Niño (Choi et al., 2013).

4. C'est-à-dire avec une anomalie chaude dans l'est du Pacifique de l'ordre de $1,25^{\circ} \mathrm{C}$, au lieu de $0,5^{\circ} \mathrm{C}$ pour les événements modérés.

\section{Synthèse}

\section{Origine d'Enso}

Pour résumer, le phénomène naturel Enso naît de la combinaison de plusieurs éléments : (1) la très grande taille du bassin Pacifique qui implique un important effet mémoire de l'océan et une forte connexion entre des points relativement éloignés du globe, (2) la nature de la dynamique équatoriale liée aux variations de la force de Coriolis et au fort couplage entre l'océan et l'atmosphère, (3) le contexte des forçages saisonnier et atmosphérique. L'ensemble de ces éléments explique l'état instable du Pacifique et donc l'existence même d'Enso. Le troisième point explique davantage l'irrégularité d'Enso et le fait que ses phases se présentent sous la forme d'événements El Niño et La Niña verrouillés sur le cycle annuel.

\section{Théorie d'Enso}

L'origine d'Enso et ses mécanismes sont aujourd'hui bien documentés. La synthèse qui en est donnée ici n'est qu'un aperçu de la complexité de la théorie et des processus en jeu. Les mécanismes invoqués pour expliquer le fonctionnement d'Enso sont souvent regroupés selon différents points de vue. Par exemple, certains voient Enso comme une oscillation auto-entretenue qui serait perturbée par les changements de saison et les aléas atmosphériques, d'autres comme une succession d'événements indépendants déclenchés aléatoirement. Ces conceptions mises à part, les mécanismes détaillés dans cet article ne sont pas exclusifs, bien qu'il n'y ait pas de certitudes sur ceux qui dominent dans la nature, d'autant que cela peut varier d'un événement à l'autre.

\section{Diversité d'Enso}

Dans la nature, chaque événement El Niño ou La Niña est unique, de par son intensité, son évolution et ses signatures spatiales (Capotondi et al., 2015). Les événements peuvent être déclenchés de différentes façons, atteindre différentes amplitudes, et tout cela dépendra de facteurs tels que l'état initial du Pacifique tropical ou la nature de l'élément déclencheur. On peut alors aisément comprendre que chaque événement ait sa propre identité et soit difficile à catégoriser. Par exemple, des débats sont en cours sur la pertinence de classer ou non les événements El Niño en deux types principaux (les «EP » pour Eastern Pacific et les « $\mathrm{CP} »$ pour Central Pacific, aussi appelés « Modokis »)

Enso continue d'agiter la communauté scientifique. Par exemple, sa prévisibilité reste limitée à environ 6 mois du fait de son caractère chaotique et de sa dépendance aux aléas atmosphériques. Sa réponse potentielle au réchauffement climatique fait également débat. Quant au fonctionnement même du phénomène, il est encore difficile d'évaluer l'importance relative des différents processus dans un événement El Niño ou dans le mode de variabilité Enso en général. Il n'en demeure pas moins vrai que nous savons pourquoi ce phénomène existe et pourquoi il est si imprévisible.

\section{Bibliographie}

Battisti D.S., 1988. Dynamics and thermodynamics of a warming event in a coupled tropical atmosphere-ocean model. J. Atmos. Sci., 45, 2889-2919. doi: 10.1175/1520-0469(1988)045<2889:DATOAW > 2.0.C0;2

Battisti D.S., Hirst A.C., 1989. Interannual variability in a tropical atmosphere-ocean model: Influence of the basic state, ocean geometry and nonlinearity. J. Atmos. Sci., 46, 1687-1712. doi: 10.1175/1520-0469(1989)046 < 1687:IVIATA > 2.0.C0;2

Belamari S., Redelsperger J., Pontaud M., 2005. Rôle des coups de vent d'ouest dans le déclenchement d’El Niño. La Météorologie, 49 , 28-37.

Bjerknes J., 1969. Atmospheric teleconnections from the equatorial Pacific. Mon. Weather Rev., 97, 163-172. doi: 10.1175/1520-0493(1969)097<0163:ATFTEP>2.3.C0;2 Boulanger J., 2003. Le phénomène climatique El Niño/Oscillation Australe (ENSO) ou Étude de mécanismes autour de l'événement El Niño 1997-1998. Habilitation à diriger des recherches, Université Pierre-et-Marie-Curie, Paris.

Burgers G., Stephenson D.B., 1999. The "normality" of El Niño. Geophys. Res. Lett., 26, 1027-1030. doi: 10.1029/1999GL900161

Capotondi A., Wittenberg A.T., Newman M., Di Lorenzo E., Yu J., Braconnot P., Cole J., Dewitte B., Giese B., Guilyardi E., Jin F., Karnauskas K., Kirtman B., Lee T., Schneider N., Xue Y., Yeh S., 2015. Understanding ENSO diversity. Bull. Am. Meteorol. Soc., 96, 921-938. doi: 10.1175/BAMS-D-13-00117.1

Chang P., Ji L., Li H., Flügel M., 1996. Chaotic dynamics versus stochastic processes in El Niño-Southern Oscillation in coupled ocean-atmosphere models. Physica D: Nonlin. Phen., 98, 301-320. doi: 10.1016/0167-2789(96)00116-9

Chang P., Battisti D.S., 1998. The physics of El Niño. Physics World, 8, 41-47.

Chen D., Lian T., Fu C., Cane M.A., Tang Y., Murtugudde R., Song X., Wu 0., Zhou L., 2015. Strong influence of westerly wind bursts on El Niño diversity. Nat. Geosci., 8 , 339-345. doi: 10.1038/ngeo2399

Choi K., Vecchi G.A., Wittenberg A.T., 2013. ENSO transition, duration and amplitude asymmetries: Role of the nonlinear wind stress coupling in a conceptual model. J. Clim., 26, 10-1175. doi: 10.1175/JCLI-D-13-00045.1 
Clarke A.J., 2008. An introduction to the dynamics of El Niño and the Southern Oscillation. Elsevier, 324 p.

Delcroix T., 1998. Observed surface oceanic and atmospheric variability in the tropical Pacific at seasonal and ENSO timescales: A tentative overview. J. Geophys. Res., 103, 18611-18633. doi: 10.1029/98JC00814

Dijkstra H.A., Burgers G., 2002. Fluid dynamics of El Niño variability. Annu. Rev. Fluid Mech., 34, 531-558. doi: 10.1146/annurev.fluid.34.090501.144936

Gill A.E., 1985. Elements of coupled ocean-atmosphere models for the Tropics. In: Elsevier Oceanography Series. Elsevier, 303-327.

Gill A.E., 1980. Some simple solutions for heat-induced tropical circulation. O. J. R. Meteorol. Soc., 106, 447-462. doi: 10.1002/qj.49710644905

Gill A.E., Clarke A.J., 1974. Wind-induced upwelling, coastal currents and sea-level changes. Deep-Sea Res. , 21, 325-345. doi: 10.1016/0011-7471(74)90038-2

Harrison D.E., Schopf P.S., 1984. Kelvin-wave-induced anomalous advection and the onset of surface warming in El Niño events. Mon. Weather Rev., 112, 923-933. doi: 10.1175/1520-0493(1984)112<0923:KWIAAA > 2.0.C0;2

Hirst A.C., 1988. Slow instabilities in tropical ocean basin-global atmosphere models. J. Atmos. Sci., 45, 830-852. doi: 10.1175/1520-0469(1988) $045<0830$ :SIITOB $>2.0$. CO;2

Hirst A.C., 1986. Unstable and damped equatorial modes in simple coupled ocean-atmosphere models. J. Atmos. Sci., 43, 606-632. doi: 10.1175/1520-0469(1986)043<0606:UADEMI>2.0.CO;2

Im S., An S., Kim S.T., Jin F., 2015. Feedback processes responsible for El Niño-La Niña amplitude asymmetry. Geophys. Res. Lett., 42, 5556-5563. doi: 10.1002/2015GL064853

Jin F., 1997. An equatorial ocean recharge paradigm for ENSO. Part I: Conceptual model. J. Atmos. Sci., 54, 811-829. doi: 10.1175/1520-0469(1997) $054<0811$ :AEORPF $>2.0 . \mathrm{CO} ; 2$

Kessler W.S., 2002. Is ENSO a cycle or a series of events? Geophys. Res. Lett., 29, 2125. doi: 10.1029/2002GL015924

Kessler W.S., McPhaden M.J., 1995. Oceanic equatorial waves and the 1991-93 El Niño. J. Clim., 8, 1757-1774. doi: 10.1175/1520-0442(1995) $008<1757: 0 E W A T E>2.0 . C 0 ; 2$

Kessler W.S., Kleeman R., 2000. Rectification of the Madden-Julian Oscillation into the ENSO cycle. J. Clim., 13, 3560-3575. doi: 10.1175/1520-0442(2000) $013<3560:$ ROTMJ0 $>2.0 . C 0: 2$

Larkin N.K., Harrison D.E., 2002. ENSO warm (El Niño) and cold (La Niña) event life cycles: Ocean surface anomaly patterns, their symmetries, asymmetries, and implications. J. Clim. , 15, 1118-1140. doi: 10.1175/1520-0442(2002)015<1118:EWENOA > 2.0.CO;2

Lengaigne M., Boulanger J., Menkes C., Spencer H., 2006. Influence of the seasonal cycle on the termination of El Niño events in a coupled general circulation model. J. Clim., 19, 1850-1868. doi: 10.1175/JCLI3706.1

Lengaigne M., Guilyardi É., Boulanger J., Menkes C., Delecluse P., Inness P., Cole J., Slingo J., 2004. Triggering of El Niño by westerly wind events in a coupled general circulation model. Clim. Dyn., 23, 601-620. doi: 10.1007/s00382-004-0457-2

L'Heureux M., 2015. An overview of the El Niño-Southern Oscillation (ENSO) since 2014. Science and Technology Infusion Climate Bull., NOAA. Disponible à l'adresse www.nws.noaa.gov/ost/climate/STIP/

Lighthill M.J., 1969. Dynamic response of the Indian Ocean to onset of the Southwest monsoon. Phil. Trans. R. Soc. A, 265, 45-92. doi: 10.1098/rsta.1969.0040

Matsuno T., 1966. Quasi-geostrophic motions in the equatorial area. J. Meteorol. Soc. Jpn, 44, 25-43.

McPhaden M.J., 1999. Genesis and evolution of the 1997-98 El Niño. Science, 283, 950-954. doi: 10.1126/science.283.5404.950

Meinen C.S., McPhaden M.J., 2000. Observations of warm water volume changes in the equatorial Pacific and their relationship to El Niño and La Niña. J. Clim., 13, 3551-3559. doi: 10.1175/1520-0442(2000)013<3551:00WWVC > 2.0.CO;2

Menkes C.E., Lengaigne M., Vialard J., Puy M., Marchesiello P., Cravatte S., Cambon G., 2014. About the role of Westerly Wind Events in the possible development of an El Niño in 2014. Geophys. Res. Lett., 41, 6476-6483. doi: 10.1002/2014GL061186

Moore A.M., Kleeman R., 1999. Stochastic forcing of ENSO by the intraseasonal oscillation. J. Clim., 12, 1199-1220. doi: 10.1175/1520-0442(1999) 012<1199:SFOEBT > 2.0.CO;2

Neelin J.D., Battisti D.S., Hirst A.C., Jin F., Wakata Y., Yamagata T., Zebiak S.E., 1998. ENSO theory. J. Geophys. Res., 103, 14261-14290. doi: 10.1029/97JC03424

Neelin J.D., 1991. The slow sea surface temperature mode and the fast-wave limit: Analytic theory for tropical interannual oscillations and experiments in a hybrid coupled model. J. Atmos. Sci., 48, 584-606. doi: 10.1175/1520-0469(1991)048<0584:TSSSTM>2.0.C0;2

Okumura Y.M., Deser C., 2010. Asymmetry in the duration of El Niño and La Niña. J. Clim., 23, 5826-5843. doi: 10.1175/2010JCLI3592.1

Penland C., Sardeshmukh P.D., 1995. The optimal growth of tropical sea surface temperature anomalies. J. Clim., 8, 1999-2024. doi: 10.1175/1520-0442(1995) $008<1999:$ TOGOTS $>2.0$. CO;2

Philander S.G., Fedorov A.A., 2003. Is El Niño sporadic or cyclic? Annu. Rev. Earth Planet. Sci., 31, 579-594. doi: 10.1146/annurev.earth.31.100901.141255

Philander S.G., 1985. El Niño and La Niña. J. Atmos. Sci., 42, 2652-2662. doi: 10.1175/1520-0469(1985)042<2652:ENALN>2.0.C0;2

Philander S.G., 1989. El Niño, La Niña, and the Southern Oscillation. Academic Press, 293 p.

Philander S.G., Yamagata T., Pacanowski R.C., 1984. Unstable air-sea interactions in the Tropics. J. Atmos. Sci., 41, 604-613. doi: 10.1175/1520-0469(1984) $041<0604:$ UASIIT $>2.0 . \mathrm{CO} ; 2$

Philip S., van Oldenborgh G.J., 2009. Significant atmospheric nonlinearities in the ENSO cycle. J. Clim., 22, 4014-4028., doi: 10.1175/2009JCLI2716.1

Picaut J., Masia F., Du Penhoat Y., 1997. An advective-reflective conceptual model for the oscillatory nature of the ENS0. Science, 277, 663-666. doi: 10.1126/ science. 277.5326 .663

Picaut J., Delcroix T., 1995. Equatorial wave sequence associated with warm pool displacements during the 1986-1989 El Niño-La Niña. J. Geophys. Res., 100, $18393-18408$. doi: 10.1029/95JC01358

Picaut J., loualalen M., Menkes C., Delcroix T., McPhaden M.J., 1996. Mechanism of the zonal displacements of the Pacific warm pool: Implications for ENSO. Science, 274, 1486-1489. doi: 10.1126/science.274.5292.1486

Rasmusson E.M., Carpenter T.H., 1982. Variations in tropical sea surface temperature and surface wind fields associated with the Southern Oscillation/El Niño. Mon. Weather Rev., 110, 354-384. doi: 10.1175/1520-0493(1982)110<0354:VITSST > 2.0.CO;2

Roulston M.S., Neelin J.D., 2000. The response of an ENSO model to climate noise, weather noise and intraseasonal forcing. Geophys. Res. Lett., 27, 723-3726. doi: 10.1029/2000GL011941

Spencer H., 2004. Role of the atmosphere in seasonal phase locking of El Niño. Geophys. Res. Lett., 31. doi: 10.1029/2004GL021619

Suarez M.J., Schopf P.S., 1988. A delayed action oscillator for ENSO. J. Atmos. Sci., 45, 3283-3287. doi: 10.1175/1520-0469(1988)045<3283:ADAOFE>2.0.CO;2

Takayabu Y.N., Iguchi T., Kachi M., Shibata A., Kanzawa H., 1999. Abrupt termination of the 1997-98 El Niño in response to a Madden-Julian Oscillation. Nature, 402, 279-282. doi: $10.1038 / 46254$ 
van Oldenborgh G.J., 2000. What caused the onset of the 1997-1998 El Niño? Mon. Weather Rev., 128, 2601-2607. doi: 10.1175/1520-0493(2000)128<2601:WCTO0T>2.0.C0;2 Vecchi G.A., 2006. The termination of the 1997-98 El Niño. Part II: Mechanisms of atmospheric change. J. Clim., 19, 2647-2664. doi: 10.1175/JCLI3780.1 Vecchi G.A., Harrison D.E., 2006. The termination of the 1997-98 El Niño. Part I: Mechanisms of oceanic change. J. Clim., 19, 2633-2646. doi: 10.1175/JCLI3776.1 Vecchi G.A., Harrison D.E., 2000. Tropical Pacific sea surface temperature anomalies, El Niño, and equatorial Westerly Wind Events. J. Clim., 13, 1814-1830. doi: 10.1175/1520-0442(2000)013<1814:TPSSTA > 2.0.C0;2

Wang B., 1995. Transition from a cold to a warm state of the El Niño-Southern Oscillation cycle. Meteorol. Atmos. Phys., 56, 17-32. doi: 10.1007/BF01022519

Wang C., Weisberg R.H., 1996. Stability of equatorial modes in a simplified coupled ocean-atmosphere model. J. Clim., 9, 3132-3148. doi: 10.1175/1520-0442(1996)009 $<3132: S O E M I A>2.0 . C 0 ; 2$

Wang C., 2001. A unified oscillator model for the El Niño-Southern Oscillation. J. Clim., 14, 98-115. doi: 10.1175/1520-0442(2001)014<0098:AUOMFT>2.0.C0;2

Wang C., Picaut J., 2004. Understanding ENSO physics - A review. Dans : Wang C., Xie S.P., Carton J.A., rédacteurs. Earth's Climate. American Geophysical Union, $21-48$. doi: 10.1029/147GM02

Weisberg R.H., Wang C., 1997. A Western Pacific oscillator paradigm for the El Niño-Southern Oscillation. Geophys. Res. Lett., 24, 779-782. doi: 10.1029/97GL00689 Zebiak S.E., Cane M.A., 1987. A model El Niño-Southern Oscillation. Mon. Weather Rev., 115, 2262-2278. doi: 10.1175/1520-0493(1987)115<2262:AMEN0>2.0.C0;2 